\title{
RESTRICCIÓN DEL CRECIMIENTO INTRAUTERINO COMO FACTOR DE RIESGO PARA MALFORMACIONES CONGÉNITAS
}

\author{
Julio Nazer H. ${ }^{1}$, Lucía Cifuentes . $^{2}$, Constanza Ramírez R. ${ }^{a}$, Camila Seymour M. ${ }^{a}$, \\ Alfredo Aguila . $^{1}$, Pilar Ureta L. ${ }^{3}$, Loreto Moya $C .^{b}$ \\ 1 Unidad de Neonatología, Maternidad del Hospital Clínico Universidad de Chile. ${ }^{2}$ Programa de Genética Humana, \\ Instituto de Ciencias Biomédicas, Facultad de Medicina, Universidad de Chile. ${ }^{3}$ Programa de Posgrado, Servicio de \\ Genética, Hospital Clínico, Universidad de Chile.
}

\footnotetext{
a Alumna, Facultad de Medicina, Universidad de Universidad de Chile.

b Alumna, Facultad de Medicina, Universidad Andrés Bello.
}

\section{RESUMEN}

Antecedentes: La restricción del crecimiento intrauterino (RCIU) se estima que está presente en el $5 \%$ de los nacimientos y es la manifestación de procesos aberrantes que impiden el desarrollo normal del feto. Objetivos: Estimar la frecuencia de esta patología en la maternidad del Hospital Clínico de la Universidad de Chile. Obtener la tasa prevalencia al nacimiento de malformaciones congénitas (MFC) y comparar la frecuencia en recién nacidos pequeños (PEG) con los adecuados (AEG) y grandes (GEG) para la edad gestacional. Método: Se estudian todos los nacimientos, vivos y mortinatos, ocurridos entre enero de 1997 a diciembre de 2008, contenidos en la base de datos del ECLAMC (Estudio Colaborativo Latino Americano de Malformaciones Congénitas) desde 1969 a la fecha. Se excluyen los recién nacidos con malformaciones como hidrocefalia, anencefalia e hidrops, que por sus características dificultan la posibilidad de clasificación en PEG, AEG o GEG. Resultados: 10,1\% de los nacimientos del período eran PEG. Entre los nacidos vivos $10 \%$ fueron $P E G$, mientras que $33,5 \%$ de los mortinatos eran $P E G(p<0,05)$. Eran malformados el $12,9 \%$ de los PEG, $8,5 \%$ de los AEG y $9,3 \%$ de los GEG $(p<0,05)$. La tasa global de malformaciones fue de $9,5 \%$; en NV el $9,4 \%$ y en mortinatos el $33 \%$. Conclusión: El RCIU es un factor que aumenta el riesgo de mortalidad fetal tardía y de presentar malformaciones congénitas.

\section{PALABRAS CLAVE: Restricción del crecimiento intrauterino, mortalidad fetal tardía, malformacio- nes congénitas}

\section{SUMMARY}

Background: Fetal growth restriction (FGR) is the result of anomalies that prevent the normal development of the fetus, it is present in about the $5 \%$ of births. Objectives: To estimate the frequency of FGR in the Clinical Hospital of the University of Chile. To estimate the congenital malformation prevalence rate at birth and compare it among small (SGE), adequate (AGE) and large (LGE) newborns according their gestational age. Methods: All live births and stillbirths included in the ECLAMC (Estudio Colaborativo Latino Americano de Malformaciones Congénitas) registered from January 1997 and December 2008 were considered. Newborns with congenital malformations that modified per se the size of the child, like hydrocephaly anencephaly and hydrops were excluded. Results: $10.1 \%$ of newborns were SGE. Among live births $10 \%$ were SGE 
instead of the $33.5 \%$ found in stillbirths $(p<0.05)$. Congenital malformation rate at birth was $12.9 \%$ in SGE, $8.5 \%$ in AGE and $9.3 \%$ in LGE newborns $(p<0.05)$. The global congenital malformation prevalence rate at birth was $9.5 \% ; 9.4 \%$ in live newborns and $33 \%$ in stillbirth. Conclusion: The FGR increase the risk of late fetal mortality and congenital anomalies.

\section{KEY WORDS: Fetal growth restriction, late fetal mortality, congenital malformations}

\section{INTRODUCCIÓN}

El peso bajo de nacimiento es un problema importante de Salud Pública. Las causas de sus dos formas, prematuridad y restricción del crecimiento intrauterino (RCIU) han sido ampliamente estudiadas.

La RCIU, el retardo del crecimiento postnatal, junto al retardo mental, las muertes fetales y las malformaciones congénitas (MC), están consideradas dentro del amplio grupo de los defectos del desarrollo. Si estimamos que el $5 \%$ de todos los recién nacidos $(\mathrm{RN})$ presentan algún grado de $\mathrm{RCIU}$, podemos comprobar que este defecto del desarrollo es más frecuente que las $\mathrm{MC}$, que se estima en alrededor del $3 \%$ y más frecuente que la mortinatalidad, que en Chile es inferior a 1\% (1).

Los tres tipos de desarrollo fetal anormal, RCIU, muerte fetal y $\mathrm{MC}$ son de fácil diagnóstico al nacimiento, y por no sufrir un subregistro importante, son apropiados para estudiarlos buscando causas o factores de riesgo determinantes para ellos.

La restricción del crecimiento intrauterino (RCIU) es la manifestación común de procesos aberrantes que impiden el crecimiento normal del feto. Muchos de estos procesos han sido dilucidados, mientras que otros permanecen sin una clara explicación (2). Complejos mecanismos genéticos y ambientales de origen materno, fetal y placentario están involucrados en este fenómeno $(2,3,4,5,6)$.

El bajo peso de nacimiento $(<2.500 \mathrm{~g})$ y la condición de pequeño para la edad gestacional se asocian con un aumento de la mortalidad perinatal, morbimortalidad infantil a corto y largo plazo que incluye un mayor riesgo de enfermedades cardiovasculares y metabólicas $(6,7,8)$. Es por esto que la prevención de la restricción del crecimiento fetal y el bajo peso de nacimiento, cuando es posible, es de gran importancia clínica y económica. Comprender la etiología es el primer paso para la prevención (7).

Dentro de las causas de origen fetal para la RCIU encontramos: las aneuploidías, la disomía uniparental e impronta genómica y las malformaciones fetales. Existe una fuerte asociación entre restricción de crecimiento intrauterino, aberraciones cromosómicas y malformaciones congénitas $(9,10)$. Fetos con alteraciones cromosómicas, incluidas las trisomías 13, 18 y 21, presentan frecuentemente restricción de su crecimiento. Aproximadamente el $90 \%$ de los fetos con trisomía 18 presentan RCIU, mientras que en trisomía 21 se asocia en el 30\% de ellos (3). Además, en crecimientos subóptimos se describen en muchas anomalías autosómicas como duplicaciones, deleciones y cromosomas en anillo (9).

Tradicionalmente el $7 \%$ de las RCIU se atribuían a aneuploidías, sin embargo, porcentajes mayores han sido reportados desde centros de referencia (3). Por ejemplo, en un centro, se identificaron defectos cromosómicos en alrededor del $20 \%$ de los fetos estudiados por RCIU entre las semanas 17 a 39 de gestación (11), y este porcentaje sería aún mayor si la falla del crecimiento se detecta antes de la semana 26 de gestación $(9,11)$. La RCIU encontrada en etapas tempranas de la gestación se asocia a un mayor riesgo de trisomía 13 y 18 (12). Por otro lado, anomalías cromosómicas se encuentran en alrededor del $7 \%$ de los recién nacidos con RCIU, lo que corresponde a diez veces de lo encontrado en niños adecuados para la edad gestacional (7).

La disomia uniparental (DUP) es la herencia de dos cromosomas homólogos desde un mismo origen parental. Aunque poco frecuente, la DUP tanto de cromosomas autosómicos como del cromosoma $X$ se ha asociado a la RCIU. La DUP materna del cromosoma 16 es el ejemplo más citado en relación a la RCIU (3).

La anomalía en la impronta genómica puede condicionar fenotipos alterados, incluyendo RCIU y defectos congénitos $(3,10)$. Dentro de esta causa encontramos dos ejemplos clásicos; el síndrome de Prader Willi y el síndrome de Silver-Russell. La pérdida de la función de genes sometidos a impronta del alelo paterno de la región 15q11-13, ocasiona el síndrome de Prader-Willi caracterizado por deficiencia en el crecimiento in útero además de otros defectos congénitos. El síndrome de Silver-Russell se caracteriza por la deficiencia en el crecimiento pre y posnatal, además de otras dismorfias. Aproximadamente en el $10 \%$ de los niños con síndrome de Silver-Russell se demuestra una DUP materna 
en genes del cromosoma 7 sometidos a impronta (3).

Un estudio poblacional conducido por el CDC de Estados Unidos demostró que más del $22 \%$ de los niños con malformaciones congénitas tienen RCIU, con un riesgo relativo de 2,6 (13). Las malformaciones fetales múltiples aumentan el riesgo de RCIU, es así como $20 \%$ de los fetos con dos malformaciones presentan RCIU, mientras que este porcentaje aumenta a $90 \%$ cuando el feto presenta nueve o más defectos (3). La presencia de una arteria umbilical única, incluso en ausencia de otras malformaciones, se asocia también a RCIU (3).

El objetivo de esta comunicación es estimar en neonatos nacidos en la maternidad del Hospital Clínico de la Universidad de Chile la frecuencia de RCIU y de MC, y la frecuencia de MC en RCIU comparados con recién nacidos sin RCIU.

\section{PACIENTES Y MÉTODO}

El análisis incluye a todos los RN vivos y mortinatos de 500 gramos o más, nacidos en el período comprendido entre el 1 de enero de 1997 y el 31 de diciembre de 2008 en la maternidad del Hospital Clínico de la Universidad de Chile.

La maternidad del Hospital Clínico de la Universidad de Chile ingresó en 1969 al Estudio Colaborativo Latino Americano de Malformaciones Congénitas (ECLAMC) (14) y se ha mantenido en él hasta la fecha. Se incluye en el registro a todos los $\mathrm{RN}$ vivos y mortinatos de peso de nacimiento de 500 gramos o más que presenten una o más malformaciones congénitas. Todos los RN son examinados por un pediatra neonatólogo de la maternidad. Se completa una ficha precodificada con los antecedentes (diagnóstico, peso de nacimiento, sexo, tipo de parto, etc.) de todo RN malformado de acuerdo con el Manual Operacional del ECLAMC. Se consignan además datos demográficos aportados por la madre en relación a edad materna y paterna, escolaridad de ambos, tipo de trabajo y otros antecedentes maternos del embarazo, como enfermedades agudas del primer trimestre, enfermedades crónicas, medicamentos usados, metrorragia en ese periodo, abortos y mortinatos previos, otros malformados en la familia. Se toma como control al $\mathrm{RN}$ de igual sexo y sin malformaciones, nacido a continuación del malformado. Los pesos de nacimientos se consideraron en gramos y la edad gestacional en semanas a contar de la fecha de la última menstruación segura y confiable, o por ecografía precoz. En este estudio no consideramos aquellos $\mathrm{RN}$ malformados que presentaban algún defecto que, de por si, podrían aumentar o disminuir el peso de nacimiento, como anencefalia, hidrocefalia e hidrops y por lo tanto alterar su catalogación.

Los RN se agruparon en 4 series, de acuerdo a su peso y edad gestacional en: término (peso adecuado para 38 semanas o más), prematuros (peso adecuado para menos de 38 semanas), RCIU (peso bajo para la edad gestacional o PEG) y recién nacidos con peso alto para la edad gestacional (GEG). Se calculó la frecuencia de malformaciones congénitas en cada uno de estos grupos. La frecuencia de distribución de cada factor de riesgo fue analizado separadamente y la categoría que tenia menor riesgo fue tomado como grupo de referencia. Se utilizó la prueba de chi cuadrado $\left(\chi^{2}\right)$ para la comparación de frecuencias, considerando diferencia significativa un valor $p<0,05$.

\section{RESULTADOS}

En el período estudiado ocurrieron 23.271 nacimientos, 23.095 nacidos vivos (NV) y 176 mortinatos (NM), lo que representa una mortinatalidad de $0,76 \%$. Hubo 2.357 RN con RCIU (10,1\%), 18.806 $(80,8 \%)$ AEG y 2.018 (8,7\%) GEG. El 10\% de los recién nacidos vivos eran PEG, mientras que $33,5 \%$ de los mortinatos tenían esa condición $(p<0,01)$.

En el total de nacimientos se diagnosticó 2.219 RN con una o más MFC $(9,5 \%)$, de ellos $2.161(9,4 \%)$ fueron nacidos vivos y 58 mortinatos (58/176=33\%). Entre los RN PEG, 299 (12,7\%) presentaban una o más MFC. Entre los RN AEG hubo 1.599 con una o más malformaciones $(8,5 \%)$ y en los RN GEG se diagnosticó 196 portadores de malformaciones congénitas $(9,3 \%)$ (Tabla I).

Al excluir de nuestro material los $74 \mathrm{RN}, 63 \mathrm{NV}$ y 11 NM (16 PEG, 44 AEG y 14 GEG), que presentaban anencefalia, hidrops o hidrocefalia, quedaron 23.197 niños (Figura 1), que constituyen la muestra en análisis.

En la Figura 2 se muestra la distribución de estos 23.197 recién nacidos, en nacidos vivos y mortinatos, según la catalogación en PEG, AEG y GEG y si eran o no malformados. Se encontró 23.034 nacidos vivos y 176 mortinatos, lo que representa una mortinatalidad de 0,76\%. Entre los NV 9,9\% eran PEG, $81,1 \%$ eran AEG y $9 \%$ GEG.

En esta submuestra hubo $2.026(8,6 \%)$ RN malformados, de los que $1.983(97,8 \%)$ eran nacidos vivos y $43(2,1 \%)$ mortinatos. De los $R N$ malformados 284 eran PEG (14,0\%), 1.556 AEG $(76,8 \%)$ y 186 GEG $(9,2 \%)$.

El $11,4 \%$ de los nacidos vivos PEG eran malformados. El $8,6 \%$ de los GEG y $8,2 \%$ de los nacidos vivos eran $A E G$.

De los mortinatos el $33,3 \%$ eran malformados. 
Tabla I

DISTRIBUCIÓN DEL TOTAL DE LOS RN SEGÚN CATALOGACIÓN AEG, GEG Y PEG, FUESEN O NO MALFORMADOS

\begin{tabular}{|c|c|c|c|}
\hline & Total & Malformados & Tasa \% \\
\hline Total nacimientos & 23.271 & 2.219 & 9,5 \\
\hline Nacidos vivos & 23.095 & 2.161 & 9,4 \\
\hline Mortinatos & 176 & 58 & 33,0 \\
\hline Total PEG & 2.357 & 299 & 12,7 \\
\hline Nacidos vivos & 2.298 & 274 & 11,9 \\
\hline Mortinatos & 59 & 24 & 40,7 \\
\hline Total AEG & 18.806 & 1.599 & 8,5 \\
\hline Nacidos vivos & 18.711 & 1.577 & 8,4 \\
\hline Mortinatos & 95 & 22 & 23,2 \\
\hline Total GEG & 2.108 & 196 & 9,3 \\
\hline Nacidos vivos & 2.086 & 189 & 9,1 \\
\hline Mortinatos & 22 & 7 & 32,0 \\
\hline
\end{tabular}

Entre los 43 mortinatos malformados, $55,8 \%$ eran PEG, 27,9\% AEG y 16,3\% GEG. La distribución de malformados entre los mortinatos AEG fue $12,6 \%$ y $31,8 \%$ GEG. La tasa de MF fue $8,6 \%$ en los NV, $24,4 \%$ en los NM, $11,4 \%$ en los NV PEG y $40,7 \%$ NM PEG.

Al comparar la frecuencia de malformaciones entre los nacidos vivos PEG, AEG y GEG, encontramos un número significativamente mayor de malformados entre los RN PEG $(11,4 \%)$ que en los otros grupos ( $8,6 \%$ en los GEG y $8,2 \%$ en los AEG) $\quad\left(\alpha^{2}=23,2 ; p<0,0001\right)$, entre los que no hay diferencias entre si. Al hacer esta comparación en los mortinatos también encontramos una diferencia significativa $\left(\chi^{2}=10,84 ; p=0,004\right)$ debido a que hay muchos más malformados entre los nacidos muertos $P E G$ que en los $A E G$, la situación en los GEG es intermedia.

Si comparamos la distribución de PEG, AEG y GEG entre nacidos vivos y mortinatos encontramos una diferencia altamente significativa $\left(\chi^{2}=95,71 ; p<\right.$ 0,000001 ), debido a que hay muchos más PEG entre los nacidos muertos que en vivos, lo mismo ocurre con los GEG pero en mucho menor magnitud.

Al revisar los diagnósticos de los mortinatos PEG encontramos que 21 de 24 niños $(87,5 \%)$ presentaban algún tipo de cromosomopatía (12 trisomías 18, 5 trisomías 13, 2 sospechas de genopatía, 1 síndrome de Turner y 1 síndrome de Cornelia de Lange (Tabla II). Entre los mortinatos AEG y GEG los diagnósticos de las malformaciones son más heterogéneas, encontramos 2 con síndrome de Down AEG y 1 síndrome de Turner.

Total

23.271

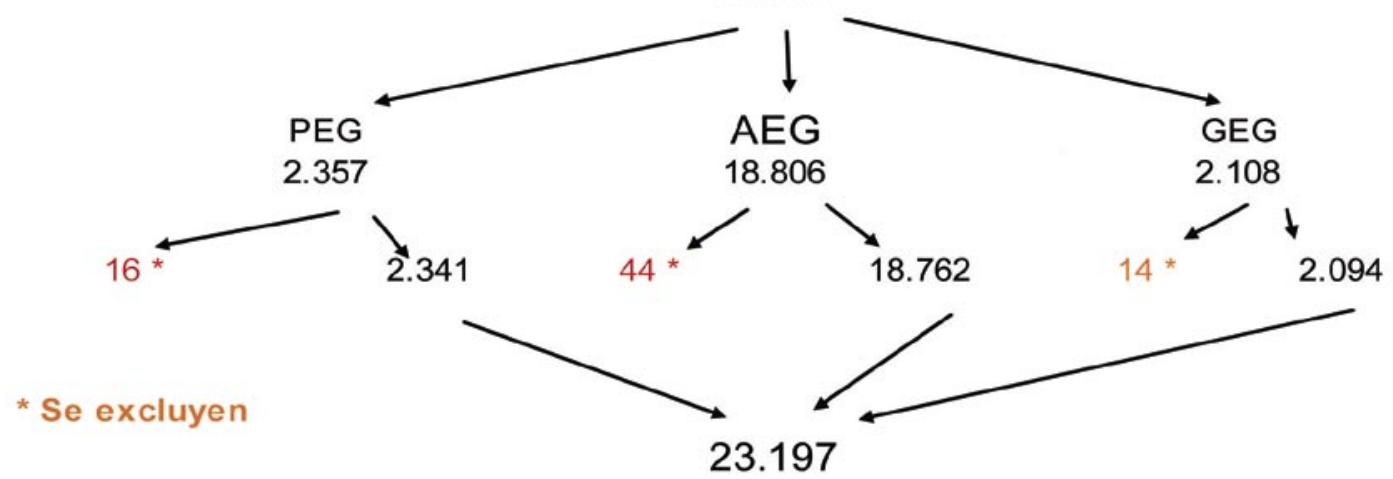

Figura 1. Distribución de los recién nacidos según fuesen PEG, AEG ○ GEG, excluyendo los casos de anencefalia, hidrocefalia e hidrops fetal. 


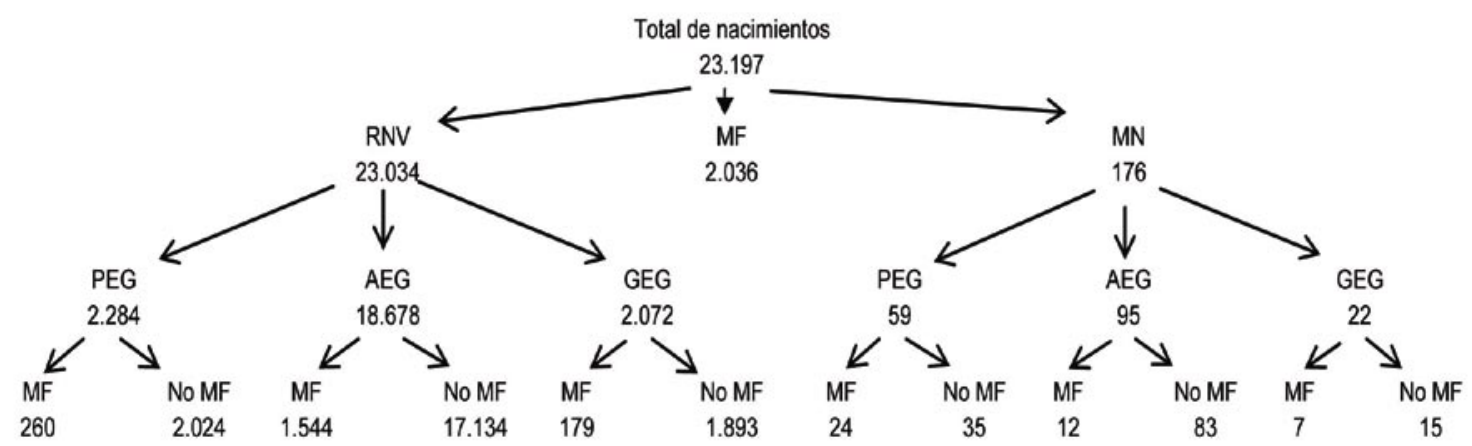

Figura 2. Distribución de los recién nacidos según sean nacidos vivos (NV) o mortinatos (MN), malformados (MF), pequeños, adecuados o grandes para la edad gestacional y malformados o no malformados.

\section{Tabla II \\ DIAGNÓSTICOS DE LOS NIÑOS MALFORMADOS}

\begin{tabular}{|c|c|c|c|}
\hline & PEG & AEG & GEG \\
\hline Trisomía 18 & 12 & & \\
\hline Trisomía 13 & 5 & & \\
\hline Síndrome de Turner & 1 & 1 & \\
\hline Síndrome de Down & & 3 & \\
\hline $\begin{array}{l}\text { Sospecha de } \\
\text { cromosomopatía }\end{array}$ & 2 & & \\
\hline Cardiopatía congénita & 1 & 1 & 2 \\
\hline $\begin{array}{l}\text { Síndrome de } \\
\text { Cornelia de Lange }\end{array}$ & 1 & & \\
\hline Agenesia renal bilateral & 2 & & \\
\hline Pie bott & & 1 & 1 \\
\hline Sexo ambiguo & & 1 & \\
\hline Displasia esquelética & & 1 & \\
\hline Gastrosquisis & & 1 & \\
\hline Mielomeningocele & & 1 & \\
\hline Onfalocele & & & 1 \\
\hline Quiste pararenal & & 1 & \\
\hline Lipoma del cuello & & & 1 \\
\hline Megavejiga & & & 1 \\
\hline Tumor del mediastino & & & 1 \\
\hline Total & 24 & 12 & 7 \\
\hline
\end{tabular}

\section{DISCUSIÓN}

La restricción del crecimiento intrauterino es un problema frecuente que se presentó en el 10\% de los nacimientos, pero este porcentaje se elevó a $33,5 \%$ entre los mortinatos. En estos últimos el $12,9 \%$ de los RN PEG fueron malformados, mientras que sólo $8,5 \%$ de los AEG y $9,3 \%$ de los GEG presentaban una o más malformaciones congénitas, diferencia estadísticamente significativa. Se destaca que el $11,4 \%$ de los nacidos vivos PEG eran portadores de una o más malformaciones congénitas.

La restricción del crecimiento fetal está condicionada por una dificultad por parte del feto para poder alcanzar su potencial de desarrollo genéticamente determinado. Antes de la introducción de la ultrasonografía como método diagnóstico en la obstetricia, era muy difícil realizar una buena medición del crecimiento intrauterino del feto. Esta nueva arma diagnóstica revolucionó los métodos de evaluación del crecimiento fetal y se desarrollaron estándares para detectar el crecimiento subnormal del feto.

Es interesante relacionar el hecho de ser un $\mathrm{RN}$ con RCIU con factores negativos a los que se asocia. Por ejemplo, en relación a mortalidad fetal, en la muestra total estudiada la mortalidad fetal tardía fue de $0,76 \%$, que se puede considerar como similar a la de USA y Canadá y muy inferior a la latinoamericana que está sobre el $2 \%$. Pues bien, el hecho que el $33,5 \%$ de los mortinatos fueran PEG y sólo el $10 \%$ de los NV fueran tuvieran esa condición $(p<0,05)$ señala que el ser $P E G$ es un factor de riesgo de muerte fetal. En segundo lugar, la asociación con malformaciones congénitas, observamos que la tasa global de MFC encontrada en toda la 
muestra $(9,5 \%)$, si bien es muy superior a las tasas nacionales, es concordante con la encontrada en otras oportunidades $(17,18)$ en la maternidad del Hospital Clínico de la Universidad de Chile. En esta muestra la tasa de MFC en recién nacidos AEG y GEG están dentro de estos márgenes. Por el contrario, la tasa de MFC en recién nacidos $P E G$ de $12,7 \%$ ( $11,4 \%$ en NV y $40,7 \%$ en mortinatos) son significativamente mayores $(p<0,0001)$.

Es importante resaltar el hecho que 21 de los 24 mortinatos PEG malformados $(87,5 \%)$ eran portadores de alguna cromosomopatía o genopatía, siendo la trisomía 18 (15 casos) y la trisomía 13 (5 casos) las más frecuentes, mientras que los tres casos de síndrome de Down eran AEG.

Nuestros resultados son comparables a los datos aportados por la literatura, que señalan que entre 80 y $85 \%$ de los nacidos vivos PEG no son portadores de malformaciones congénitas ni cromosomopatías (19). En un estudio sueco se encontró que el $21,1 \%$ de los $\mathrm{RN}$ con restricción del crecimiento intrauterino eran portadores de malformaciones congénitas y sólo $8,2 \%$ de aquellos cuya restricción del crecimiento era simétrico.

Es importante señalar en los fetos con anomalías cromosómicas la restricción es frecuente y puede estar ocasionada en alguna medida por una placenta insuficiente, dado que ésta también posee aberraciones cromosómicas (20), encontrándose mosaicismo placentario en algunos casos de restricción del crecimiento intrauterino (21).

\section{CONCLUSIÓN}

Nuestros resultados permiten confirmar que la $\mathrm{RCIU}$ es un factor de alto riego de mortalidad fetal tardía, que se asocia con malformaciones congénitas incompatibles con la vida y en un gran número de casos obedece a alteraciones cromosómicas o genéticas. La presencia de malformaciones congénitas de origen no cromosómico limita el potencial de crecimiento del feto por los mismos factores que han determinado la anomalía estructural.

\section{BIBLIOGRAFÍA}

1. Nazer J, Margozzini J, Rodrìguez M, Rojas M, Cifuentes L. Malformaciones invalidantes en Chile: Estudio ECLAMC 1982-1997. Rev Med Chile 2001;129:6774.

2. Maulik D, Frances Evans J, Ragolia L, Fetal Growth Restriction: Pathogenic Mechanism. Clin Obstet Gynecol 2006;49:219-27.

3. Monk D, Moore GE. Intrauterine growth restriction, genetic causes and consequences. Semin Fetal Neonatal Med 2004;9:371-8.
4. Ergaz Z, Avgil M, Ornoy A Intrauterine growth restriction - etiology and consequences: What do we know about the human situation and experimental animal models? Reprod Toxicol 2005;20:301-22.

5. Maulik D. Fetal Growth Restriction: The Etiology. Clin Obstet Gynecol 2006;49:228-35.

6. Snijders RJ, Sherrod C, Gosden CM, Nicolaides KH. Fetal growth retardation: associated malformations and chromosomal abnormalities. Am J Obstet Gynecol 1993;168:547-55.

7. Bahado-Singh RO, Lynch L, Deren O, Morroti R, Copel JA, Mahoney MJ, Williams J 3rd. First-trimester growth restriction and fetal aneuploidy: the effect of type of aneuploidy and gestational age. Am J Obstet Gynecol 1997;176:976-80.

8. Khoury MJ, Erickson JD, Cordero JF. Congenital malformations and intrauterine growth retardation: a population study. Pediatrics 1988;82:83-90.

9. Pallotto Eugenia K, Kilbride Howard W. Perinatal outcome and later implications of intrauterine growth restriction. Clin Obstet Gynecol 2006;49:257-69.

10. Fretts RC. Etiology and prevention of stillbirth. Am J Obstet Gynecol 2005;193:1923-35.

11. Maulik D. Fetal growth compromise: definitions, standards, and classification. Clin Obstet Gynecol 2006;49:214-8.

12. Cetin I, Foidart JM, Miozzo M, Raun T, Jansson T, Tsatsaris V, et al. Fetal growth restriction: a workshop report. Placenta 2004;25(8-9):753-7.

13. Boulet SL, Alexander GR, Salihu HM, Kirby RS, Carlo WA. Fetal growth risk curves: defining levels of fetal growth restriction by neonatal death risk. Am J Obstet Gynecol 2006;195:1571-7.

14. Sheridan $C$. Intrauterine growth restriction--diagnosis and management. Aust Fam Physician 2005;34:71723.

15. Pardo RA, Nazer J, Cifuentes L. Prevalencia al nacimiento de malformaciones congénitas y de menor peso de nacimiento en hijos de madres adolescentes. Rev Med Chile 2003;131:1165-72.

16. Baschat A. Pathophysiology of fetal growth restriction: implications for diagnosis and surveillance. Obstet Gynecol Survey 2004;59:617-27.

17. Nazer J, Aravena T, Cifuentes L. Malformaciones congénitas: un problema emergente. Rev Med Chile 2001;129:895-904.

18. Nazer J, Cifuentes L, Aguila A, Juárez ME, Cid MP, Godoy ML, et al. Efectos de la fortificación de la harina con ácido fólico sobre la evolución de las tasas de malformaciones congénitas en las maternidades chilenas participantes del ECLAMC. Rev Med Chile 2007;135:198-204.

19. Sheridan C. Intrauterine growth restriction -- diagnosis and management. Aust Family Physician 2005;34:71723.

20. Nikkilä A, Källén B and Marsál K. Fetal growth and congenital malformations. Ultrasound Obstet Gynecol 2007;29:289-95.

21. Kalousek DK. Current topic: confined placental mosaisism and intrauterine fetal development. Placenta 1994;15:219-30. 